\title{
Hybrid Peroral Endoscopic Myotomy for Achalasia with Prior Treatment Failure
}

\author{
In Kyung Yoo', Abdullah OzgurYeniova ${ }^{2}$ and Joo Young Cho' \\ ${ }^{1}$ Division of Gastroenterology, Department of Internal Medicine, Cha Bundang Medical Center, Seongnam, Korea, ${ }^{2}$ Division of \\ Gastroenterology, Department of Internal Medicine, Tokat Gaziosmanpaşa University, Tokat, Turkey
}

Achalasia is a neurodegenerative motility disorder caused by enteric neuron damage in the lower esophageal sphincter. Peroral endoscopic myotomy (POEM) is a standard treatment method for achalasia. Previous treatment modalities may affect the outcome of POEM as they cause submucosal fibrosis. We report a new technique called "hybrid POEM" for the treatment of patients with achalasia who had been previously treated with pneumatic balloon dilatation. We performed two techniques of POEM simultaneously, the standard POEM for the upper part of the submucosal tunnel and open POEM for the stenotic part of the esophagogastric junction. We dissected the mucosa and submucosa, and performed myotomy simultaneously. We overcame submucosal fibrosis of the esophagogastric junction, which was caused by the previous hybrid POEM treatment. The risks of mucosal incision and technical challenge of submucosal tunneling for the fibrotic area may be reduced by hybrid POEM. Clin

Endosc 2021;54:127-130

Key Words: Achalasia; Esophagus; Peroral endoscopic myotomy

\section{INTRODUCTION}

Achalasia is a neurodegenerative motility disorder caused by enteric neuron damage in the lower esophageal sphincter. Treatment modalities of achalasia include botulinum toxin injection (BTI), pneumatic dilatation (PD) and Heller myotomy. ${ }^{1}$ Peroral endoscopic myotomy (POEM) was introduced by Inoue et $\mathrm{al}^{2}$ POEM has gained popularity as a primary treatment modality for achalasia as it is effective and less invasive.

Interventions that aim to relieve symptoms of achalasia can cause damage and fibrosis in submucosal space of the lower esophageal sphincter. ${ }^{3}$ As POEM is a treatment modality that uses the submucosal area, prior treatment modalities

Received: December 27, 2019 Revised: March 9, 2020

Accepted: March 11, 2020

Correspondence: Joo Young Cho

Division of Gastroenterology, Department of Internal Medicine, Cha Bundang Medical Center, 59 Yatap-ro, Bundang-gu, Seongnam 13496, Korea Tel: +82-31-780-5641, Fax: +82-32-780-5005, E-mail: cjy6695@naver.com ORCID: https://orcid.org/0000-0001-9085-0313

(c) This is an Open Access article distributed under the terms of the Creative Commons Attribution Non-Commercial License (http://creativecommons.org/ licenses/by-nc/3.0) which permits unrestricted non-commercial use, distribution, and reproduction in any medium, provided the original work is properly cited. can influence the outcome of the POEM. ${ }^{4}$ Here we report a case where the patient experienced complication due to PD and was treated with a new technique called "hybrid POEM (H-POEM)".

\section{CASE REPORT}

A 43-year-old man with a complaint of dysphagia was admitted to another hospital 3 months ago. He was diagnosed with type I achalasia. His Eckardt score was 12, and high-resolution manometry showed high lower esophageal sphincter pressure (62 $\mathrm{mm} \mathrm{Hg}$ ), high integrated relaxation pressure (43 $\mathrm{mm} \mathrm{Hg}$ ), and aperistalsis of the esophageal body. He received PD, but pneumomediastinum occurred after PD. He was discharged after chest tube insertion and 20 days of antibiotics treatment. The patient was then referred to our hospital because of the worsening of dysphagia. Endoscopy revealed stenosis in the esophagogastric junction (EGJ) (Fig. 1); the esophagogram showed no passage of barium in the 5 minutes delayed phase (Fig. 2). 


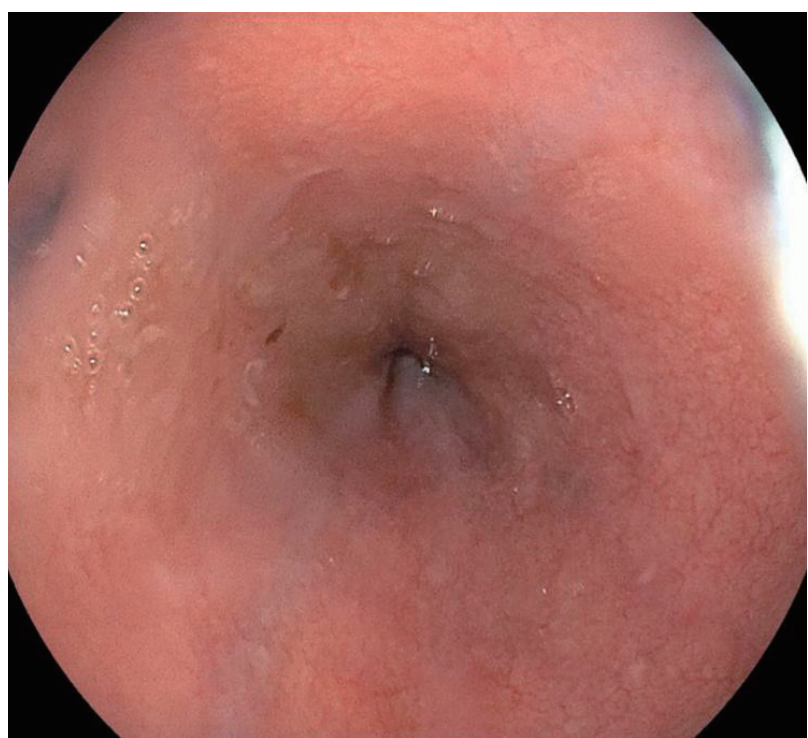

Fig. 1. Endoscopy before the procedure.

In order to overcome the stricture at EGJ due to fibrosis that occurred during the healing of the perforation, we decided to perform H-POEM. H-POEM consists two steps; standard POEM (S-POEM) for the proximal part and open POEM (O-POEM) for the stenotic part (Fig. 3, Supplementary Video 1).

After a mucosal incision $7 \mathrm{~cm}$ proximal to the EGJ stenosis, a submucosal tunnel was created as described previously. ${ }^{2} \mathrm{~A}$ guide-wire was inserted into the stomach to guide the submucosal tunneling and for inserting the stent. When we reached the stenotic part, we could not dissect the submucosal fiber, and switched to O-POEM. To remove the EGJ stenosis, mucosal and submucosal incision and myotomy were performed simultaneously. Selective myotomy was also performed in the submucosal tunnel of the proximal part of tunnel. A fully covered esophageal stent with a diameter of $18 \mathrm{~mm}$ and length of $10 \mathrm{~cm}$ (Hanarostent ${ }^{\circledR}$; M.I.Tech, Pyeongtaek, Korea) was inserted to cover the O-POEM site. Finally, the mucosal entry was closed with endoclips. The patient was discharged after two weeks with no specific complications. No stent migration occurred. The esophageal stent was removed after two months. The follow-up endoscopy and barium esophagogram revealed no stenosis (Figs. 4, 5). In the follow-up esophageal high-resolution manometry, the esophageal sphincter pressure was decreased to $30 \mathrm{~mm} \mathrm{Hg}$ from $62 \mathrm{~mm} \mathrm{Hg}$, and the integrated relaxation pressure decreased to $18 \mathrm{~mm} \mathrm{Hg}$ from 43 $\mathrm{mm} \mathrm{Hg}$.

Contractions of the esophageal body were peristaltic, but mostly weak in amplitude. The patient did not experience dysphagia during the follow-up period.

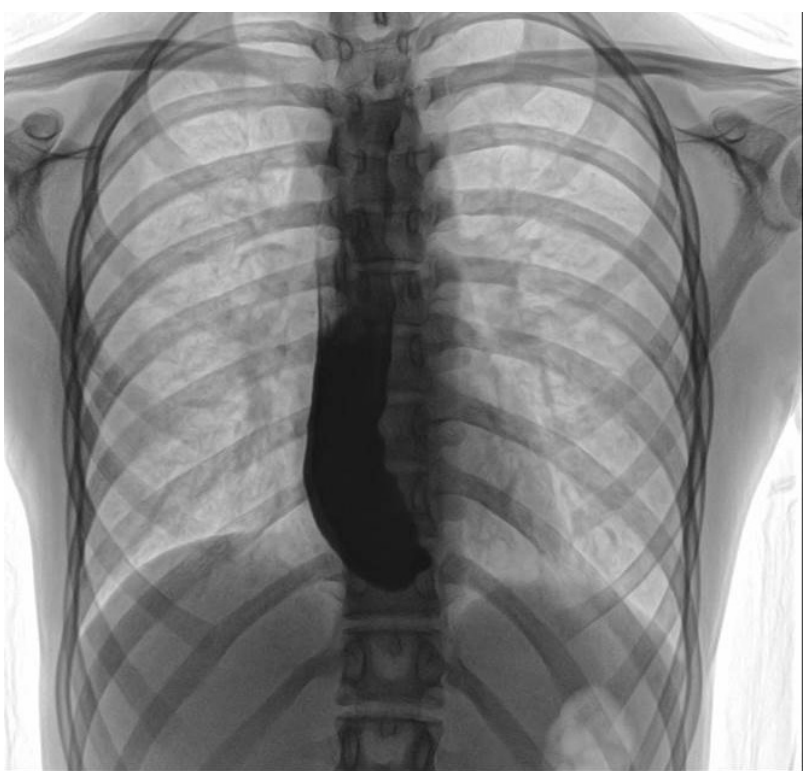

Fig. 2. Barium esophagogram before the procedure.

\section{DISCUSSION}

POEM was described a decade age ago and is considered a standard treatment modality for achalasia. The choice of other treatment modalities like BTI and PD depends on the condition of the patient. POEM may be used as the second-line treatment after failure of or complications due to BTI and PD. ${ }^{5}$

In this case study, we defined a novel method for achalasia with prior treatment failure. We defined this technique as H-POEM because we applied two techniques simultaneously. Even though POEM is described as a safe and effective method for prior treatment failure or complications, ${ }^{4}$ fibrosis and scar formation can influence the outcome of S-POEM. Some patients, especially with sigmoid-shaped achalasia and severe submucosal fibrosis, may show difficulties associated with POEM. Submucosal fibrosis may be a consequence of prior treatment; submucosal tunneling might be technically demanding and sometimes impossible. ${ }^{3} \mathrm{O}-\mathrm{POEM}$ has been described as a rescue technique for the treatment of achalasia. ${ }^{6,7}$ After submucosal injection $6-10 \mathrm{~cm}$ above the EGJ, the first mucosal incision was made $2 \mathrm{~cm}$ beyond the EGJ. After the mucosal incision, submucosal fibers were dissected and selective myotomy was performed. A case series which recruited 82 patients with achalasia showed that O-POEM is a highly effective method for treating achalasia patients with previous treatment failure. ${ }^{8}$ The authors reported that in comparison with S-POEM, O-POEM was simple and quick. No esophageal stent was used. Mucosal injury is one of the most common adverse effects of S-POEM. Mucosal incision is made ad- 

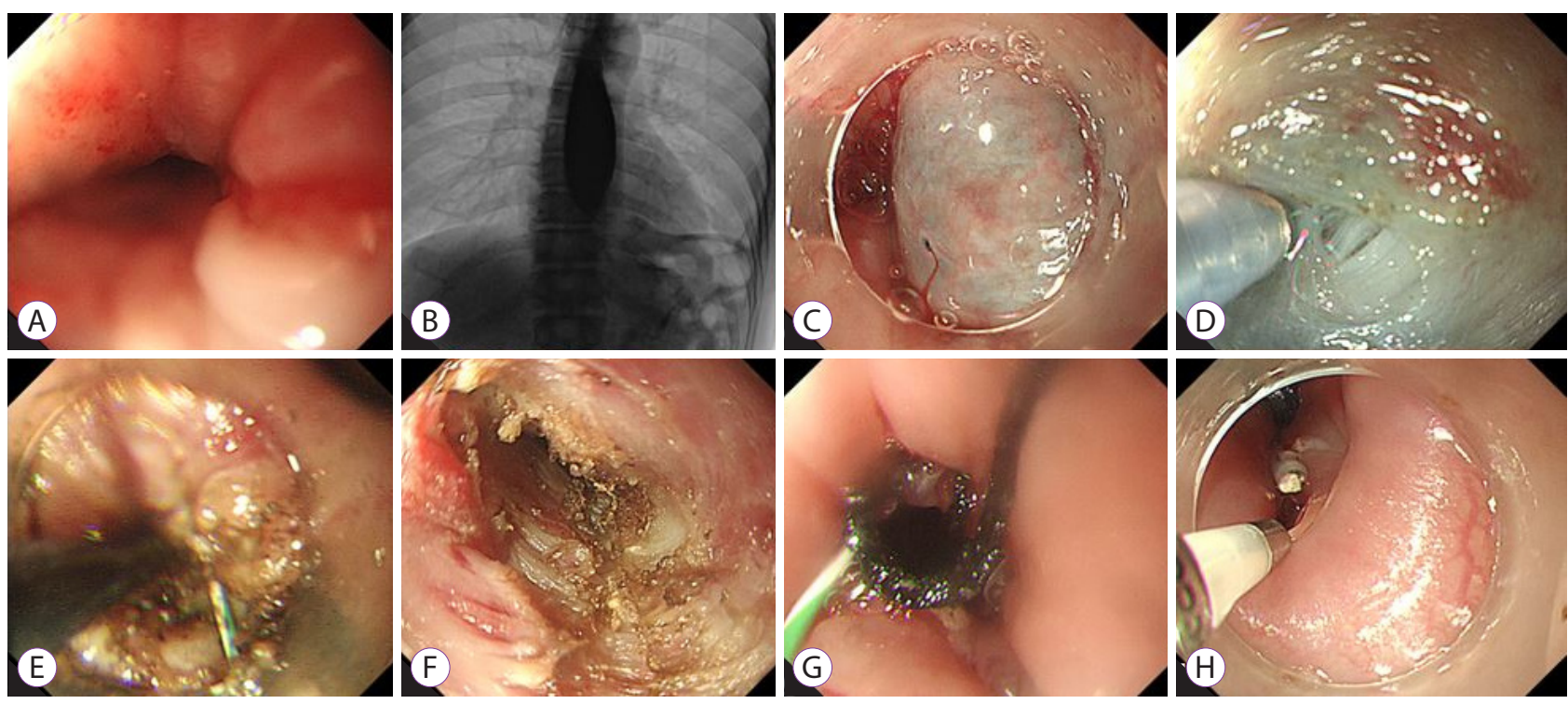

Fig. 3. Hybrid peroral endoscopic myotomy (POEM). (A) Endoscopy reveals very tight esophagogastric junction (EGJ). (B) Barium radiograph showing no passage after the 5 minute delayed phase. (C) Submucosal injection was given $7 \mathrm{~cm}$ proximal to the EGJ. (D) Careful injection and dissection on the EGJ. (E) Open POEM around the stenotic part. (F) Standard POEM in the tunnel. (G) Fully covered esophageal stent insertion. (H) Entry site clipping.



Fig. 4. Endoscopy after the procedure.

vertently during O-POEM, and so, the surgeon does not have to pay attention to mucosal incision, which is a complication of S-POEM. Although O-POEM is not defined as a standard method, it may be considered as a rescue therapy.

H-POEM may be considered as an alternative rescue therapy for challenging cases. It has benefits over O-POEM because it is a hybrid method. The surgeon can start with S-POEM, and when a technical difficulty arises because of submucosal

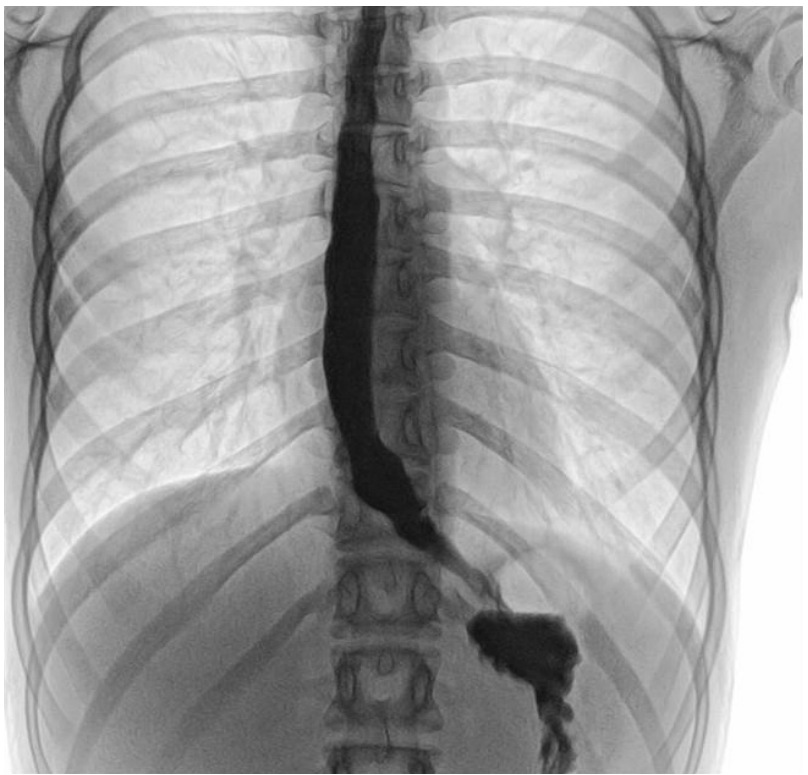

Fig. 5. Barium esophagogram after the procedure.

fibrosis, the procedure can be changed to O-POEM for the fibrotic area. Using O-POEM, the fibrotic area can be removed; a fully covered stent insertion can promote the healing of an ulcer. S-POEM may be performed in the non-fibrotic area. The risks of mucosal incision and technical challenge of submucosal tunneling in the fibrotic area may be reduced by H-POEM. 


\section{Conflicts of Interest}

Joo Young Cho has been a Senior Consultant of Clinical Endoscopy; however, he was not involved in the peer reviewer selection, evaluation, or decision process of this article. The other authors have no potential conflicts of interest.

\section{Funding}

None.

\section{ORCID}

In Kyung Yoo:

Abdullah OzgurYeniova:

Joo Young Cho:

https://orcid.org/0000-0003-0909-339X

https://orcid.org/0000-0003-1681-364X

https://orcid.org/0000-0001-9085-0313

Supplementary Material

Video 1. Hybrid peroral endoscopic myotomy as a treatment for complicated achalasia (https://doi.org/10.5946/ce.2020.013v001).

\section{REFERENCES}

1. Zaninotto G, Leusink A, Markar SR. Management of achalasia in 2019. Curr Opin Gastroenterol 2019 Apr 24 [Epub]. https://doi.org/10.1097/ MOG.0000000000000544.

2. Inoue $\mathrm{H}$, Minami $\mathrm{H}$, Kobayashi $\mathrm{Y}$, et al. Peroral endoscopic myotomy (POEM) for esophageal achalasia. Endoscopy 2010;42:265-271.

3. Wu QN, Xu XY, Zhang XC, et al. Submucosal fibrosis in achalasia patients is a rare cause of aborted peroral endoscopic myotomy procedures. Endoscopy 2017;49:736-744.

4. Nabi Z, Ramchandani M, Chavan R, et al. Peroral endoscopic myotomy in treatment-naïve achalasia patients versus prior treatment failure cases. Endoscopy 2018;50:358-370.

5. Sharata A, Kurian AA, Dunst CM, Bhayani NH, Reavis KM, Swanström LL. Peroral endoscopic myotomy (POEM) is safe and effective in the setting of prior endoscopic intervention. J Gastrointest Surg 2013;17:11881192.

6. Liu W, Wu CC, Hu B. Open peroral endoscopic myotomy for achalasia with failed Heller myotomy. Dig Endosc 2018;30:268-269.

7. Liu W, Zeng HZ, Chen HL, Wu CC, Ye LS, Hu B. Open peroral endoscopic myotomy (O-POEM) for the treatment of achalasia. Dis Esophagus 2017;30:1-2.

8. Liu W, Zeng XH, Yuan XL, et al. Open peroral endoscopic myotomy for the treatment of achalasia: a case series of 82 cases. Dis Esophagus 2019;32:1-7 